\title{
Teaching Effect Analysis Based on Function P-sets
}

\author{
Xiuqing $\mathrm{Yu}$ \\ Department of Mathematics Dezhou University, Dezhou253023, China
}

Tel: 86-139-6923-1258Ｅmail: sddzyxq@163.com

This research was supported by the Natural Science Foundation and the Education Science Foundation of Shandong Province of China (ZR2010AL019), and (2010JZ123) respectively.

\begin{abstract}
Function P-sets which is composed of function internal P-set and function outer P-set, is a function set pair and has dynamic and law characteristics. Based on function P-sets, this paper presents the concepts such as data law, $\bar{F}$ - data law, $F$-data law and bi-data law, and then $\bar{F}$-data law theorem, $F$-data law theorem, bi-data law band theorem, data law recovery theorem, bi-data law identification theorem and law identification criterion are proposed. By using these results, teaching effect analysis is obtained and applications on bilingual education are given. Function P-sets is a new method in the research of dynamic data system.
\end{abstract}

Keywords: Function P-sets, Data law, Teaching effect

\section{Introduction}

In 2008, Refs.(SHI Kai-quan. 2008)(SHI Kai-quan. 2009) originated P-sets $\left(X^{\bar{F}}, X^{F}\right) \quad$ by introducing dynamic characteristic into general set $X$.Refs. (SHI Kai-quan Shi, Li Zhang. 2009)(YU Xiu-qing. 2010) (YU Xiu-qing. 2010) (SHI Kai-quan\&Tingcheng Chang. 2005) (SHI Kai-quan \&LI Xiu-hong, 2010) discussed application in many fields. In 2010, Refs. ( ZHANG Li\&CUI Yu-quan. 2010) introduced function into P-sets, and gave concept and structure of function P-sets $\left(Q^{\bar{F}}, Q^{F}\right)$. Function P-sets are function set pair composed of function internal P-set $Q^{\bar{F}}$ and function outer $P$-set $Q^{F}$ together, they have dynamic and law characteristic. It is also to say: when functions in function set $Q$ are transferred out from $Q, Q$ becomes function internal P-set $Q^{\bar{F}}$, on the other hand,when functions out of $Q$ are transferred into $Q, Q$ becomes function outer P-set $Q^{F}$, therefore function P-sets has dynamic characteristic. Moreover because function is law, so it also has law characteristic. Based on above content and features of function P-sets, the concepts of data law, $\bar{F}$-data law, $F$-data law and bi-data law are proposed, the bi-data law band theorem, recovery theorem and identification theorem are given, and identification criterion of data law are put forward. Using these results, this paper gives teaching effect analysis modal (TEAM) and discusses application of TEAM on bilingual education in university.

To facilitate the discussion and acceptance of the results, function P-sets and its simple structure are introduced into the following section as theoretical preparation.

2. Function P-sets and its structure

Assumptions: $\quad V$ is a nonempty finite attribute universe, $\alpha$ is a nonempty finite attribute set in $V$. T $(x)$ is a nonempty finite function universe of discourse, $Q(x)=\left\{u(x)_{1}, u(x)_{2}, \cdots, u(x)_{m}\right\}$ is a finite function sets in $\mathrm{T}(x), u(x)_{i}, i=1,2,3, \ldots, m$ is a function in $Q(x), F=\left\{f_{1}, f_{2}, \ldots, f_{r}\right\}$ and $\bar{F}=\left\{\bar{f}_{1}, \bar{f}_{2}, \ldots, \bar{f}_{s}\right\}$ are function transfer families, $f \in F$ and $\bar{f} \in \bar{F}$ are function transfer. For expressing briefly and without misunderstanding, $\mathrm{T}(x)$, $Q(x)$ and $[u(x)]$ are denoted by $\mathrm{D}, Q$ and $[u]$ respectively.

Given a finite general function set $Q=\left\{u_{1}, u_{2}, \cdots, u_{m}\right\} \subset \mathrm{T}$, and $\alpha=\left\{\alpha_{1}, \alpha_{2}, \cdots, \alpha_{k}\right\} \subset V$ is the attribute set of $Q$.

$Q^{\bar{F}}$ is called function internal packet set of $Q$, called function internal P-set for short, moreover $Q^{\bar{F}}=Q-Q^{-}$,

and $Q^{-}$is called $\bar{F}$-function deleted set of $Q$, moreover

$Q^{-}=\{u \mid u \in Q, \bar{f}(u)=w \bar{\in} Q, \bar{f} \in \bar{F}\}$,

if the attribute set $\alpha^{F}$ of $Q^{\bar{F}}$ satisfies 
$\alpha^{F}=\alpha \bigcup\left\{\left|\alpha^{\prime}\right| f(\beta)=\alpha^{\prime} \in \alpha, f \in F\right\}$,

where $\beta \in V, \beta \bar{\in} \alpha, f \in F$ turns $\beta$ into $\alpha^{\prime}=f(\beta) \in \alpha$.

$Q^{F}$ is called outer function packet set of $X$, called outer P-set for short, moreover

$Q^{F}=Q \cup Q^{+}$,

and $Q^{+}$is called $F$-function supplemented set, moreover

$Q^{+}=\left\{u^{\prime} \mid w \in T, w \bar{\in} Q, f(w)=u^{\prime} \in Q, f \in F\right\}$

if the attribute set $\alpha^{\bar{F}}$ of $Q^{F}$ satisfies

$\alpha^{\bar{F}}=\alpha-\left\{\alpha_{i} \mid \bar{f}\left(\alpha_{i}\right)=\beta_{i} \bar{\in} \alpha, \bar{f} \in \bar{F}\right\}$

where, $\alpha_{i} \in \alpha, \bar{f} \in \bar{F}$ turns $\alpha_{i}$ into $\bar{f}\left(\alpha_{i}\right)=\beta_{i} \bar{\in} \alpha . \quad \alpha^{\bar{F}} \neq \phi$.

The set pair which is composed of function internal P-set $X^{\bar{F}}$ and function outer P-set $X^{F}$ is called P-sets (packet sets) generated by the general function set $Q$, briefly called P-sets, moreover

$\left(Q^{\bar{F}}, Q^{F}\right)$

where, the general set $Q$ is called the ground set of $\left(Q^{\bar{F}}, Q^{F}\right)$.

Theorem 2.1 (Dynamic characteristic theorem of function P-sets) Function $\operatorname{set} Q$, its function internal P-set $Q^{\bar{F}}$ and its function outer P-set $Q^{F}$ satisfies

$Q^{\bar{F}} \subseteq Q \subseteq Q^{F}$

Theorem 2.2 (The relation theorem between P-sets and general set) suppose $F=\left\{f_{1}, f_{2}, \cdots, f_{m}\right\}$ and $\bar{F}=\left\{\bar{f}_{1}, \bar{f}_{2}, \cdots, \bar{f}_{n}\right\}$ be function transfer families, if $\left(Q^{\bar{F}}, Q^{F}\right)$ is a function P-sets generated by a general function set $Q$, then

$$
\left(Q^{\bar{F}}, Q^{F}\right)_{F=\bar{F}=\phi}=Q
$$

In fact, if $F=\phi$, then $\left\{\alpha^{\prime} \mid f(\beta)=\alpha^{\prime} \in \alpha, f \in F\right\}=\phi$, i.e., $\alpha^{F}=\alpha$, the equation (1) changes into $Q^{\bar{F}}=Q$ and (2) changes into $Q^{-}=\{u \mid u \in Q, \bar{f}(u)=w \bar{\in} Q, \bar{f} \in \bar{F}\}=\phi$; if $\bar{F}=\phi$, then $\left\{\alpha_{i} \mid \bar{f}\left(\alpha_{i}\right)=\beta_{i} \bar{\in} \alpha, \bar{f} \in \bar{F}\right\}=\phi$, i.e., $\quad \alpha^{\bar{F}}=\alpha$, the equation (4) changes into $Q^{F}=Q$ and (5) changes into $Q^{+}=\left\{u^{\prime} \mid w \in T, w \bar{\in} Q, f(w)=u^{\prime} \in Q, f \in F\right\}=\phi$, then we have (9).

\section{Data law and its characteristics}

Definition 1 Given finite function set $Q=\left\{u_{1}, u_{2}, u_{3}, \ldots, u_{m}\right\}, \forall u_{i} \in Q$ has discrete data distributions, moreover

$y_{i}=\left(y_{i 1}, y_{i 2}, \ldots y_{i k}, \ldots y_{i n+1}\right)$

$y$ is called the compound data set of $u_{i}$, moreover

$y=\left(y_{1}, y_{2}, \ldots, y_{3}, \ldots, y_{n+1}\right)$

where, $y_{k}=\sum_{j=1}^{m} y_{j k}, k=1,2, \ldots, n+1 ; y_{i k}, y_{k} \in R^{+}, R^{+}$is nonnegative real number set, $i=1,2, \ldots, m$.

Definition $2 p(x)$ is called the data law generated by $Q$, if $p(x)$ is the polynomial function generated by data points

$\left(x_{1}, y_{1}\right),\left(x_{2}, y_{2}\right), \ldots,\left(x_{n+1}, y_{n+1}\right)$,

moreover

$p(x)=\sum_{j=1}^{n+1} y_{j} \prod_{\substack{i, j=1 \\ i \neq j}}^{n+1} \frac{x-x_{i}}{x_{j}-x_{i}}=\rho_{n} x^{n}+\rho_{n-1} x^{n-1}+\ldots+\rho_{1} x+\rho_{0}$,

where (12) is the data points constituted by(11).

Definition 3 Suppose that $Q^{\bar{F}}$ is function internal P-set of $Q, p(x)^{\bar{F}}$ is called internal data law generated by $Q^{\bar{F}}$, briefly called $\bar{F}$-data law, moreover

$p(x)^{\bar{F}}=a_{n} x^{n}+a_{n-1} x^{n-1}+\ldots+a_{1} x_{1}+a_{0}$ 
where $\forall u_{i} \in Q^{\bar{F}}, u_{i}$ has discrete data distribution $\quad y_{i}=\left(y_{i 1}, y_{i 2}, \ldots y_{i k}, \ldots y_{i n+1}\right), i=1,2, \ldots, \operatorname{card}\left(Q^{\bar{F}}\right)$. card $=$ cardinal number. $p(x)^{\bar{F}}$ is generated via formula(10)-(12).

Definition 4 Suppose that $Q^{F}$ is function outer P-set of $Q, p(x)^{F}$ is called outer data law generated by $Q^{F}$, briefly called $F$-data law, moreover

$p(x)^{F}=b_{n} x^{n}+b_{n-1} x^{n-1}+\ldots+b_{1} x_{1}+b_{0}$

Where, $\forall u_{j} \in Q^{F}, u_{j}$ has discrete data distribution $y_{i}=\left(y_{i 1}, y_{i 2}, \ldots y_{i k}, \ldots y_{i n+1}\right), i=1, \quad 2, \ldots, \operatorname{card}\left(Q^{F}\right)$. card $=$ cardinal number. $p(x)^{F}$ is generated via formula(10)-(12).

Definition 5 The data law pair composed of $p(x)^{\bar{F}}$ and $p(x)^{F}$ is call bi-data law generated by P-sets $\left(Q^{\bar{F}}, Q^{F}\right)$, written as

$\left(p(x)^{\bar{F}}, p(x)^{F}\right)$

According to definitions 1-5, the following theorems can be got.

Theorem 3.1 ( $\bar{F}$-data law theorem) If $p(x)$ is data law generated by function set $Q$, then there exists an $\bar{F}$ -data law $p(x)^{\bar{F}}$ satisfying

$$
p(x)^{\bar{F}}+\nabla p(x)^{\bar{F}}=p(x)
$$

where, $\quad \nabla p(x)^{\bar{F}} \geq 0, p(x)^{\bar{F}}$ is the data law generated by $Q^{\bar{F}}$.

Proof Assumed that $Q=\left\{u_{1}, u_{2}, \cdots, u_{m}\right\}$ is the given finite function set and $y_{i}=\left\{y_{i, 1}, y_{i, 2}, \cdots, y_{i, n+1}\right\}$ is the data set of $u_{i} \in Q$. Then there exists data set $y=\left\{y_{1}, y_{2}, \cdots, y_{n+1}\right\}=\left\{\sum_{i=1}^{m} y_{i, 1}, \sum_{i=1}^{m} y_{i, 2}, \cdots, \sum_{i=1}^{m} y_{i, n+1}\right\}$ with respect to $Q \quad$. For $Q^{\bar{F}}=\left\{u_{1}, u_{2}, \cdots, u_{m-r}\right\} \quad$, apparently, there exists $\bar{F}$-data set $y^{\bar{F}}=\left\{y_{1}^{\bar{f}}, y_{2}^{\bar{f}}, \cdots, y_{n+1}^{\bar{f}}\right\}=\left\{\sum_{i=1}^{m-r} y_{i, 1}, \sum_{i=1}^{m-r} y_{i, 2}, \cdots, \sum_{i=1}^{m-r} y_{i, n+1}\right\}$, namely there is $y_{\lambda}^{\bar{f}} \in y^{\bar{F}}$. For any $\lambda \in\{1$, $2, \cdots, n, n+1\}, \quad y_{\lambda}^{\bar{f}}=\sum_{i=1}^{m-r} y_{i, \lambda} \leq \sum_{i=1}^{m} y_{i, \lambda}=y_{\lambda}$, then there exists $\nabla \varepsilon_{\lambda} \in R^{+}$satisfying $y_{\lambda}-\nabla \varepsilon_{\lambda}=y_{\lambda}^{\bar{f}}$.By definition 2 , formula (16) is got.

Theorem 3.2 ( $F$-data law theorem) If $p(x)$ is data law generated by function set $Q$, then there exists an $F$ -data law $p(x)^{F}$ satisfying

$$
p(x)^{F}-\Delta p(x)^{F}=p(x),
$$

where, $\Delta p(x)^{F} \geq 0, p(x)^{F}$ is the data law generated by $Q^{F}$.

Theorem 3.3 ( bi-data law band theorem) If $\varphi^{\bar{F}}$ and $\varphi^{F}$ are cures generated by $p(x)^{\bar{F}}$ and $p(x)^{F}$ respectively, then

1. There exists bi-data law band, written as $\operatorname{BAN}\left\{\varphi^{\bar{F}}, \varphi^{F}\right\}$, which takes $\varphi^{\bar{F}}$ as lower boundary and takes $\varphi^{F}$ as upper boundary.

2. $p(x)^{\bar{F}}$ and $p(x)^{F}$ satisfy

$p(x)^{F}-p(x)^{\bar{F}} \geq 0$

The proof of theorem 3.3 can be easily obtained from formula (1), (4) and (13), so omitted.

Theorem 3.4 (Boundary coincidence theorem) The bi-data law band $\operatorname{BAN}\left\{\varphi^{\bar{F}}, \varphi^{F}\right\}$ satisfies

$\varphi^{\bar{F}}=\varphi^{F}$

if and only if,

$\left(Q^{\bar{F}}, Q^{F}\right)_{\bar{F}=F=\phi}=Q$.

In fact, if $\bar{F}=F=\phi$, then the expression (3) turns into $\alpha^{F}=\alpha$ and the expression (6) turns into $\alpha^{\bar{F}}=\alpha$, thus $Q^{\bar{F}}$ in (1) and $Q^{F}$ in (4) changes into $Q$, i.e., $Q^{\bar{F}}=Q^{F}=Q$. So $\varphi^{F}$ is coincident with $\varphi^{\bar{F}}$, i.e., $\varphi^{F}=\varphi^{\bar{F}}=\varphi$. So the proof of theorem 3.4 is omitted. 
Theorem 3.5 (attribute dependence theorem of $\bar{F}$-data law) suppose $\alpha_{i}^{F}, \alpha_{j}^{F}$ and $\alpha_{k}^{F}$ are attribute sets of $Q_{i}^{\bar{F}}, Q_{j}^{\bar{F}}$ and $Q_{k}^{\bar{F}}$ respectively, if they satisfy $\alpha_{i}^{F} \subseteq \alpha_{j}^{F} \subseteq \alpha_{k}^{F}$, then there exists an $\bar{F}$-data law sequence satisfying

$p(x)_{k}^{\bar{F}} \leq p(x)_{j}^{\bar{F}} \leq p(x)_{i}^{\bar{F}}$

where, $p(x)_{i}^{\bar{F}}, p(x)_{j}^{\bar{F}}$ and $p(x)_{k}^{\bar{F}} \quad$ are $\bar{F}$-data laws generated by $Q_{i}^{\bar{F}}, Q_{j}^{\bar{F}}$ and $Q_{k}^{\bar{F}}$ respectively. $Q_{i}^{\bar{F}}, Q_{j}^{\bar{F}}$ and $Q_{k}^{\bar{F}}$ are function internal P-set of function set $Q$.

Theorem 3.6 (attribute dependence theorem of $F$-data law) suppose $\alpha_{i}^{\bar{F}}, \alpha_{j}^{\bar{F}}$ and $\alpha_{k}^{\bar{F}}$ are attribute sets of $Q_{i}^{F}, Q_{j}^{F}$ and $Q_{k}^{F}$ respectively, if they satisfy $\alpha_{i}^{\bar{F}} \subseteq \alpha_{j}^{\bar{F}} \subseteq \alpha_{k}^{\bar{F}}$, then there exists an $F$-data law sequence satisfying

$p(x)_{i}^{F} \leq p(x)_{j}^{F} \leq p(x)_{k}^{F}$,

where, $p(x)_{i}^{F}, p(x)_{j}^{F}$ and $p(x)_{k}^{F} \quad$ are data laws generated by $Q_{i}^{F}, Q_{j}^{F}$ and $Q_{k}^{F}$ respectively. $Q_{i}^{F}, Q_{j}^{F}$ and $Q_{k}^{F}$ are function outer P-set of function set $Q$.

Theorem 3.7 ( $\bar{F}$-data law recovery theorem of bi-data law) Given a bi-data law $\left(p(x)^{\bar{F}}, p(x)^{F}\right)$, if $p(x)^{\bar{F}}=p(x)$, then the

attribute sets $\alpha^{F}$ and $\alpha$ with respect to $p(x)^{\bar{F}}$ and $p(x)$ respectively satisfy

$\alpha^{F}-\left\{\alpha_{i} \mid \alpha_{i} \in \alpha^{F}, \bar{f}\left(\alpha_{i}\right)=\beta_{i} \bar{\in} \alpha^{F}\right\}=\alpha$.

Proof Suppose $y=\left\{y_{1}, y_{2}, \cdots, y_{n+1}\right\}$ and $y^{\bar{F}}=\left\{y_{1}^{\bar{f}}, y_{2}^{\bar{f}}, \cdots, y_{n+1}^{\bar{f}}\right\}$ are compound data sets of $Q=\left\{u_{1}\right.$, $\left.u_{2}, \cdots, u_{m}\right\}$ and $Q^{\bar{F}}=\left\{u_{1}, u_{2}, \cdots, u_{m-r}\right\}$ respectively. From the expressions (1)-(3), we know there exist attribute sets $\alpha^{F}$ and $\alpha$ with respect to $Q^{\bar{F}}$ and $Q$ respectively satisfying $\alpha \subseteq \alpha^{F}$. Obviously, there exist some attributes $\alpha_{i}$ in $\alpha^{F}$, if they are deleted from it, i.e., $\alpha^{F}-\left\{\alpha_{i} \mid \alpha_{i} \in \alpha^{F}, \bar{f}\left(\alpha_{i}\right)=\beta_{i} \bar{\in} \alpha^{F}\right\}=\alpha$, $Q^{\bar{F}}$ is recovered to $Q$, then $p(x)^{\bar{F}}=p(x)$.

Theorem 3.8 ( $F$-data law recovery theorem of bi-data law) Given a bi-data law $\left(p(x)^{\bar{F}}, p(x)^{F}\right)$, if $p(x)^{F}=p(x)$, then the

attribute sets $\alpha^{\bar{F}}$ and $\alpha$ with respect to $p(x)^{F}$ and $p(x)$ respectively satisfy

$\alpha^{\bar{F}} \cup\left\{\beta_{i} \mid \beta_{i} \in V, \beta_{i} \bar{\in} \alpha^{\bar{F}}, f\left(\beta_{i}\right)=\alpha_{i} \in \alpha^{\bar{F}}\right\}=\alpha$

Its proof is similar to the theorem 3.7, so omitted.

\section{Teaching effect analysis}

In this section $p(x)=\rho_{n} x^{n}+\rho_{n-1} x^{n-1}+\ldots+\rho_{1} x+\rho_{0}$ is data law generated by function set $Q$, $p(x)^{\bar{F}}=a_{n} x^{n}+a_{n-1} x^{n-1}+\ldots+a_{1} x_{1}+a_{0} \quad \bar{F}$-data law generated by function internal P-set $Q^{\bar{F}}$ of $Q$ and $p(x)^{F}=b_{n} x^{n}+b_{n-1} x^{n-1}+\ldots+b_{1} x_{1}+b_{0} \quad F$ - data law generated by function outer P-set $Q^{F}$ of $Q$.

Definition $5 d^{\bar{F}}$ is called $\bar{F}$-measurement degree, moreover

$d^{\bar{F}}=\sqrt{\sum_{i=0}^{n}\left(a_{i}-p_{i}\right)^{2}}$

Definition $6 d^{F}$ is called $F$-measurement degree, moreover

$d^{F}=\sqrt{\sum_{i=0}^{n}\left(b_{i}-p_{i}\right)^{2}}$

Definition 7 The number pair composed by $d^{\bar{F}}$ and $d^{F}$ is called bi-measurement degree, denoted as $\left(d^{\bar{F}}, d^{F}\right)$ 
Theorem 4.1 (Identification theorem of bi-data laws) If $\left(p(x)_{i}^{\bar{F}}, p(x)_{i}^{F}\right)$ and $\left(p(x)_{j}^{\bar{F}}, p(x)_{j}^{F}\right)$ are bi-data laws generated by $\left(Q_{i}^{\bar{F}}, Q_{i}^{F}\right)$ and $\left(Q_{j}^{\bar{F}}, Q_{j}^{F}\right)$ of $Q$ respectively, $\left(d_{i}^{\bar{F}}, d_{i}^{F}\right)$ and $\left(d_{j}^{\bar{F}}, d_{j}^{F}\right)$ are their bi-measurement degree respectively, moreover,

$d_{i}^{\bar{F}}=d_{j}^{\bar{F}}, d_{i}^{F}=d_{j}^{F}$

then the two bi-data law cannot be identified, denoted by

$$
\mathrm{UNI}\left(\left(p(x)_{i}^{\bar{F}}, p(x)_{i}^{F}\right),\left(p(x)_{j}^{\bar{F}}, p(x)_{j}^{F}\right)\right)
$$

where, $\mathrm{UNI}$ is brief expression of unidentification.

Theorem 4.2 If $\left(p(x)_{i}^{\bar{F}}, p(x)_{i}^{F}\right)$ and $\left(p(x)_{j}^{\bar{F}}, p(x)_{j}^{F}\right)$ are bi-data laws generated by function P-sets $\left(Q_{i}^{\bar{F}}, Q_{i}^{F}\right)$ and $\left(Q_{j}^{\bar{F}}, Q_{j}^{F}\right)$ of $Q$ respectively, $\left(d_{i}^{\bar{F}}, d_{i}^{F}\right)$ and $\left(d_{j}^{\bar{F}}, d_{j}^{F}\right)$ are their bi-measurement degree respectively, moreover,

$d_{i}^{\bar{F}} \geq d_{j}^{\bar{F}}$

then

$$
z\left(\alpha_{i}^{+}\right) \geq z\left(\alpha_{j}^{+}\right)
$$

Where, $z\left(\alpha_{i}^{+}\right)$and $z\left(\alpha_{j}^{+}\right)$are important degree of attribute sets $\alpha_{i}^{+}$and $\alpha_{j}^{+}$to function set $Q$ respectively, $\alpha_{i}^{+}$ and $\alpha_{j}^{+}$are supplement attribute sets of $\alpha$, i.e., $\alpha_{i}^{+}=\left\{\alpha_{i}{ }^{\prime} \mid \beta \in V, \beta \bar{\in} \alpha, f(\beta)=\alpha_{i}{ }^{\prime} \in \alpha\right\}$, $\alpha_{j}^{+}=\left\{\alpha_{j}{ }^{\prime} \mid \beta \in V, \beta \bar{\in} \alpha, f(\beta)=\alpha_{j}^{\prime} \in \alpha\right\} . \alpha$ is attribute set of $Q$.

Theorem 4.3 If $\left(p(x)_{i}^{\bar{F}}, p(x)_{i}^{F}\right)$ and $\left(p(x)_{j}^{\bar{F}}, p(x)_{j}^{F}\right)$ are bi-data laws generated by function P-sets $\left(Q_{i}^{\bar{F}}, Q_{i}^{F}\right)$ and $\left(Q_{j}^{\bar{F}}, Q_{j}^{F}\right)$ of $Q$ respectively, $\left(d_{i}^{\bar{F}}, d_{i}^{F}\right)$ and $\left(d_{j}^{\bar{F}}, d_{j}^{F}\right)$ are their bi-measurement degree respectively, moreover,

$d_{i}^{F} \leq d_{j}^{F}$

Then

$z\left(\alpha_{i}^{-}\right) \geq z\left(\alpha_{j}^{-}\right)$

Where, $z\left(\alpha_{i}^{-}\right)$and $z\left(\alpha_{j}^{-}\right)$express important degree of attribute sets $\alpha_{i}^{-}$and $\alpha_{j}^{-}$to function set $Q$ respectively, $\alpha_{i}^{-}$and $\alpha_{j}^{-}$are deleted attribute sets of $\alpha \quad$ i.e., $\quad \alpha_{i}^{-}=\left\{\alpha_{i} \mid \alpha_{i} \in \alpha, f\left(\alpha_{i}\right)=\beta_{i} \bar{\epsilon} \alpha\right\}$ $\alpha_{j}^{-}=\left\{\alpha_{j} \mid \alpha_{j} \in \alpha, f\left(\alpha_{j}\right)=\beta_{j} \bar{\epsilon} \alpha\right\}$.

\section{Identification criterion of bi-data law}

If the attribute sets $\left(\alpha_{i}^{F}, \alpha_{i}^{\bar{F}}\right)$ of $\left(p(x)_{i}^{\bar{F}}, p(x)_{i}^{F}\right)$ and $\left(p(x)_{j}^{\bar{F}}, p(x)_{j}^{F}\right)$ satisfy $\left(\alpha_{i}^{F}, \alpha_{i}^{\bar{F}}\right)-\left(\alpha_{j}^{F}, \alpha_{j}^{\bar{F}}\right) \neq \phi$, then $\left(p(x)_{i}^{\bar{F}}, p(x)_{i}^{F}\right)$ and $\quad\left(p(x)_{j}^{\bar{F}}, p(x)_{j}^{F}\right)$ can be identified, denoted as

$\operatorname{IDE}\left(\left(p(x)_{i}^{\bar{F}}, p(x)_{i}^{F}\right),\left(p(x)_{j}^{\bar{F}}, p(x)_{j}^{F}\right)\right)$

here the expression $\left(\alpha_{i}^{F}, \alpha_{i}^{\bar{F}}\right)-\left(\alpha_{j}^{F}, \alpha_{j}^{\bar{F}}\right) \neq \phi$ means both $\alpha_{i}^{F}-\alpha_{j}^{F} \neq \phi$ and $\alpha_{i}^{\bar{F}}-\alpha_{j}^{\bar{F}} \neq \phi$. IDE is brief expression of identification.

The following gives teaching effect analysis, for teaching process is similar to function P-sets.

There is teaching effect function set, denoted as $Q=\left\{u_{1}, u_{2}, \cdots, u_{m}\right\}, \alpha$ is the attribute set of $Q$, $y_{i}=\left\{y_{i 1}, y_{i 2}, y_{i 3}, \ldots, y_{i n+1}\right\}$ means number value set of $u_{i} \in Q, u_{i}$ is a discrete function ,which influences teaching effect, $y_{i k} \in R^{+}, k=1,2,3, \ldots, n+1, R^{+}$is the positive real number set, then they form $y=\left\{y_{1}, y_{2}, \cdots, y_{n+1}\right\}=\left\{\sum_{i=1}^{m} y_{i, 1}, \sum_{i=1}^{m} y_{i, 2}, \cdots, \sum_{i=1}^{m} y_{i, n+1}\right\}$ by formula (11) on interval $[a, b]$. Here $p(x)$ is the teaching effect law on continuous interval $[a, b]$ by formula(13), As a matter of fact, when some unfavorable factors(attribute elements of $Q$ ) from teachers, students and other aspects are deleted from $\alpha$, the class teaching effect expands its scale, that is to say, $y$ expanded into $y^{\prime}$, then $y$ changed into 
$y^{\prime}=\left\{y_{1}^{\prime}, y_{2}^{\prime}, \cdots, y_{m+r}^{\prime}\right\}=\left\{\sum_{i=1}^{m+r} y_{i, 1}^{\prime}, \sum_{i=1}^{m+r} y_{i, 2}^{\prime}, \cdots \sum_{i=1}^{m+r} y_{i, n+1}^{\prime}\right\} ;$ it is easy to get $p(x)^{F}>p(x), d^{F}>0$, and $z\left(\alpha^{-}\right)>0$. When some unfavorable factors (attribute elements of $Q$ ) are transferred into $\alpha$, the class teaching effect shrinks, that is to say, $y$ shrunk into $y^{\prime \prime}=\left\{y_{1}, y_{2}, \cdots, y_{n+1}\right\}$, $y^{\prime \prime}=\left\{y_{1}^{\prime \prime}, y_{2}^{\prime \prime}, \cdots, y_{n+1}^{\prime \prime}\right\}=\left\{\sum_{i=1}^{m-\lambda} y_{i, 1}^{\prime \prime}, \sum_{i=1}^{m-\lambda} y_{i, 2}^{\prime \prime}, \cdots, \sum_{i=1}^{m-\lambda} y_{i, n+1}^{\prime \prime}\right\} ;$ it is easy to get $p(x)^{\bar{F}}<p(x), d^{\bar{F}}>0$, and $z\left(\alpha^{+}\right)>0$. This fact is very simple and can be accepted by anybody.

In teaching effect analysis system, a effect distribution at a certain period (for example, a term)are generally presented, as a matter of fact, this distribution is a cure $p(x)$ at interval $[a, b]$, here $y_{k}$ is the teaching effect value at point $k, y_{k} \in R^{+}$. The effect distribution is actually a predicted value, it changes along with the factors in many aspects. When there is a good teaching environment, $y_{k} \in y$ turns into $y_{k}^{\prime}=y_{k}+\Delta \sigma_{k}$, here $\Delta \sigma_{k}$ is the added value of effect value $y_{k}$ at point $k$, so the cure $p(x)^{\prime}$ and $p(x)$ satisfy $p(x)^{\prime}>p(x)$; When there is a bad teaching environment, $y_{k} \in y$ turns into $y_{k}^{\prime \prime}=y_{k}-\nabla \varepsilon_{k}$, here $\nabla \varepsilon_{k}$ is the added value of effect value $y_{k}$ at point $k$, so the cure $P(x)^{\prime \prime}$ and $p(x)$ satisfy $p(x)^{\prime \prime}<p(x)$; thus $P(x)$ " and $p(x)^{\prime}$ form $\operatorname{BAN}\left\{p(x)^{\prime \prime}, p(x)^{\prime}\right\}$ and $p(x)$ is just in it. This is the real situation of teaching effect analysis system every teacher must meet.

\section{Application of bilingual education}

Freshmen are divided into three classes according to their entrance grades randomly. No.1 is chosen as compared class. No. 2 and No. 3 are experiment classes. Advanced mathematics of the three classes are taught by the same teacher. But No1 is taught in native language, No2 mainly in native language including little English professional nouns and little simply sentences, No.3 almost in English only including explaining in Chinese. By using knowledge in section 3 and section4, table1 is got.

Table1 four teaching effect compound data of each class in the first term(data has be dealt with.)

\begin{tabular}{llccc} 
Test number & 1 & 2 & 3 & 4 \\
\hline$y$ in No.1 (Compared class) & 1.22 & 1.01 & 0.62 & 0.61 \\
\hline$y^{F}$ in No.2 & 1.52 & 1.29 & 1.15 & 1.09 \\
$y^{\bar{F}}$ in No.3 & & & & 0.02 \\
\hline
\end{tabular}

According to section 4 and formula(13), the three data law curves of teaching effect are obtained, they are $p_{1}(x)=-0.597 x^{3} \quad+4.855 x^{2}-12.098 x+8.92 \quad, \quad p_{2}(x)=-1.473 x^{3}+11.375 x^{2}-24.894 x+17.33 \quad, \quad$ and $p_{3}(x)=-0.922 x^{3}+7.275 x^{2}-17.223 x+12.09$ respectively, moreover $p_{3}(x) \leq p_{1}(x) \leq p_{2}(x)$. This is fit to real fact. In No.1 students not only met no difficulty, but also had more learning interest than before during learning. In No3 students met more difficulty in understanding what the teacher taught for teacher gave a lesson almost in English, consequently they lose interest to learn and become no self-confidence. This teaching effect analysis shows the necessity of adjusting teaching modal.

\section{Discussion}

Refs. ( ZHANG Li\&CUI Yu-quan. 2010) originated the concept and structure of function P-sets. Based on function P-sets, the concept of bi-data law was proposed and the characteristics of bi-data law were discussed in this paper. Bi-data law exists in many kinds of information system, but it does not attract more attention now. In fact, the bi-data law reveals the value characteristics of dynamic information systems. From the discussion and application given in this paper, we can think of that function P-sets is a new theory and a new method in the research of dynamic information system.

\section{References}

LIN Hong-kang\&LI Yu-ying. (2010). P-sets and its P-separation theorems. An International Journal Advances in Systems Science and Applications, 24 -5-251.

SHI Kai-quan \&LI Xiu-hong, (2010). Camouflaged information and its on identification and its applications. An International Journal Advances in Systems Science and Applications, 208-216.

SHI Kai-quan Shi, Li\&Zhang. (2009). Internal P-sets and data outer-recovery. Journal of Shandong University(Natural Science), 8-14. 
SHI Kai-quan\&Tingcheng Chang. (2005). One direction S-rough sets. International Journal of Fuzzy Mathematics, 2005, 13(2):319-334.

SHI Kai-quan. (2008). P-sets. Journal of Shandong University(Natural Science), 77- 84.

SHI Kai-quan. (2009). P-sets and its applications. Advances in Systems Science and Applications, 168-178.

YU Xiu-qing. (2010). Dynamic characteristics of P-sets. Computer Engineering and Applications, 45-48.

YU Xiu-qing. (2010). Recognition and screening of P-sets. Journal of Shandong University(Natural Science), 94-98.

ZHANG Guan-yu\&LI En-zhong. (2010). Information gene and its information knock-out/knock-in. An International Journal Advances in Systems Science and Applications, 267-275.

ZHANG Li\&CUI Yu-quan. (2010). Outer P-sets and data internal-recovery. An International Journal Advances in Systems Science and Applications, 229-236. 